\title{
Graphene Activation Explains the Enhanced Hydrogen Evolution on Graphene-Coated Molybdenum Carbide Electrocatalysts
}

\author{
Timothy T. Yang ${ }^{1}$ and Wissam A. Saidi ${ }^{*}$ \\ ${ }^{1}$ Department of Materials Science and Engineering, University of Pittsburgh, Pittsburgh, PA 15260, \\ United States \\ *alsaidi@pitt.edu
}

\section{Computational Details}

We use the Vienna Ab Initio Simulation Package (VASP) for the first-principles density functional theory (DFT) calculations. We use the Perdew-Burke-Ernzerhof (PBE) exchange-correlational functional to solve the Kohn-Sham equations within periodic boundary conditions, and the PAW pseudopotentials to describe electron-nucleus interactions. ${ }^{1-4}$ We include Tkatchenko and Scheffler van der Waals corrections for all calculations ${ }^{5}$. The electronic self-consistent loops are terminated within energy-change tolerance of $1 \times 10^{-}$ ${ }^{6} \mathrm{eV}$. The climbing image nudged elastic band method is applied for finding minimum energy pathways with the tolerance of $0.05 \mathrm{eV} / \AA^{6}{ }^{6}$

To model the HER mechanisms in acidic solution, we use a standard setup for solid-liquid interface by employing a single water monolayer located at $\sim 3 \AA$ above surface to replicate bulk water. ${ }^{7}$ In the water monolayer, each $\mathrm{H}_{3} \mathrm{O}^{+}$is bonded with three $\mathrm{H}_{2} \mathrm{O}$ in Eigen structure. ${ }^{8}$ Starting from an ice-like configuration, similar to what was done for $\mathrm{Pt}$ (111) with proton concentration of $1 / 8,{ }^{9}$ we find that the $\mathrm{H}_{2} \mathrm{O}$ molecules can be in three different configurations such that their $\mathrm{H}$ atoms point toward (H-down) or away (H-up) from the surface, or their molecular planes are in parallel to the surface (H-flat). The most stable water layer corresponds to half of the $\mathrm{H}_{2} \mathrm{O}$ molecules in $\mathrm{H}$-down and the other half in $\mathrm{H}$-flat configuration, and a less stable water layer is also found by turning $1 / 4 \mathrm{H}_{2} \mathrm{O}$ from $\mathrm{H}$-down to $\mathrm{H}$-up configuration. We verified that the different water configurations have a small effect of less than $0.1 \mathrm{eV}$ on the activation barriers of the HER elementary steps.

\section{Atomic Structure Models}

In DFT calculations, the relaxed $\gamma$-phase MoC in $P \overline{6} m 2$ hexagonal structure (WC-type) has the lattice parameters of $a=b=2.89 \AA$ and $c=2.81 \AA$, in agreement with experiments. ${ }^{10,}{ }^{11}$ The $\gamma$-MoC (001) surface is chosen to couple with graphene as it has been verified to be a stable termination with low surface energy that is exposed on $\gamma-\mathrm{MoC}$ nanoparticles ${ }^{12-14}$. The periodic heterostructure slab models are constructed using $4 \times 4$ single graphene supported by $2 \sqrt{3} \times 2 \sqrt{3}$ MoC-(001). The $\gamma$-MoC supported graphene is constructed using Zur and McGill scheme whereby the interface translational symmetry is compatible with the symmetry on both sides of the interface. ${ }^{15}$ The interface separation between the $\gamma$-MoC Mo-terminated (001) and graphene is $2.2 \AA$. The models are composed of 5-8 atomic layers with a $14 \AA$ vacuum to minimize the interactions between images in non-periodic direction. We fixed bottom 2-4 layers at bulk positions, and allowed all the other coordinates to relax with a tolerance of $1 \times 10^{-6} \mathrm{eV}$. We use $2 \times 2 \times 1$ Monkhorst-Pack k-point to sample the Brillion zone and planewave cutoff of $400 \mathrm{eV}$. For the supported graphene with defects, we have verified that there is no surface reconstruction by doubling the surface supercell. 

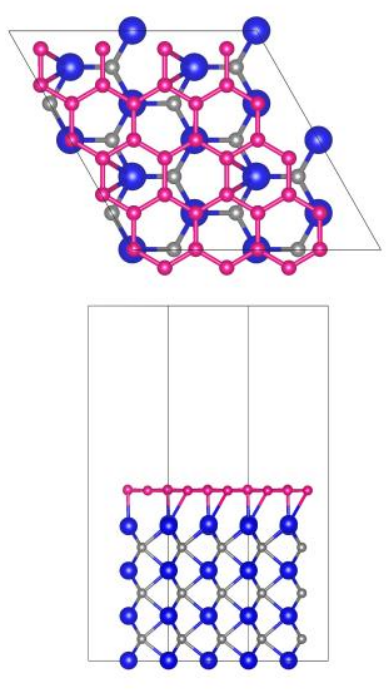

MoC-G
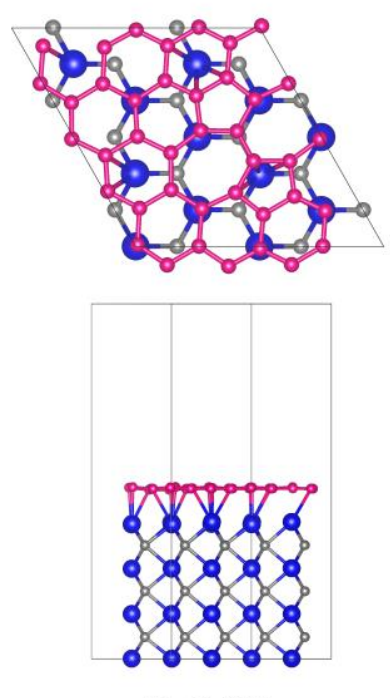

MoC-SW
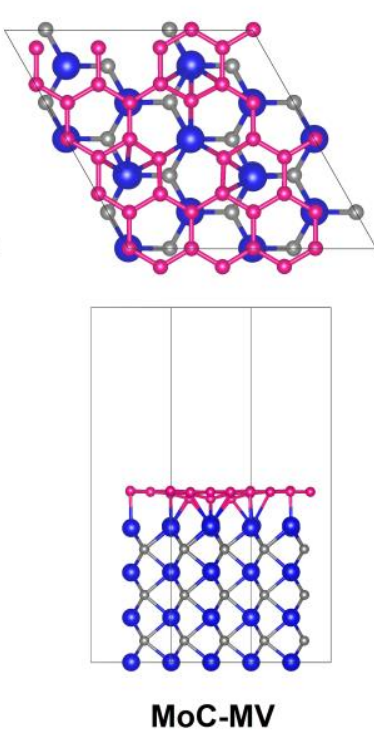

MoC-MV
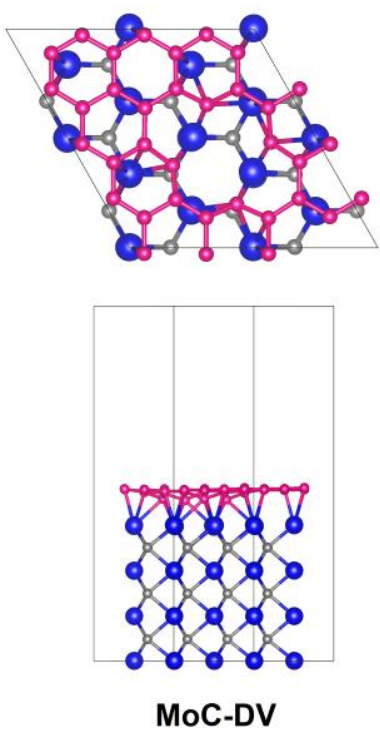

Figure S1. The structure of $\psi-M o C$ supported graphene systems (top and side views). The carbon atoms of graphene $(\gamma-\mathrm{MoC})$ are in magenta (gray), and the Mo atoms of $\psi$-MoC are in blue.

\section{Adhesion energy}

To quantify the structure stability of $\gamma$-MoC (001) Mo-terminated supported graphene, we compute the adhesion energy defined as

$$
E_{\text {adh }}=\frac{E_{\gamma-\mathrm{MoC} / \text { graphene }}-E_{\gamma-\mathrm{Moc}}-E_{\text {graphene }}}{A},
$$

where $E_{\gamma-\mathrm{MoC} / \text { graphene }}, E_{\gamma-\mathrm{MoC}}$ and $E_{\text {graphene }}$ are the DFT energies of the $\gamma$-MoC (001) Mo-terminated supported graphene system, $\gamma$-MoC (001) Mo-terminated surface and unsupported graphene, respectively, and $A$ is the contacting surface area between the two isolated interfaces. The adhesion energy of MoC/graphene systems are summarized in Table S1.

Table S1. The adhesion energies $E_{a d h}$ of $\gamma$-MoC (001) Mo-terminated systems. We also show the vertical distance between graphene and $\gamma-\mathrm{MoC}(001)$ in addition to graphene rumpling.

\begin{tabular}{lccc}
\hline Systems & $\begin{array}{c}\boldsymbol{E}_{\text {adh }} \\
\left(\mathbf{J} / \mathbf{m}^{\mathbf{2}}\right)\end{array}$ & $\begin{array}{c}\text { Vertical Distance } \\
(\AA)\end{array}$ & $\begin{array}{c}\text { Graphene Rumpling } \\
(\AA)\end{array}$ \\
MoC-G & -1.73 & 2.12 & 0.54 \\
MoC-SW & -1.93 & 2.12 & 0.39 \\
MoC-MV & -2.78 & 2.18 & 0.05 \\
MoC-DV & -2.82 & 2.18 & 0.09
\end{tabular}

Table S1 shows that the adhesion energy of the $\gamma$-MoC Mo-terminated (001) supported graphene is -1.73 $\mathrm{J} / \mathrm{m}^{2}$ that is about five times larger than that of $\mathrm{SiO}_{2} /$ graphene $^{16}\left(-0.35 \mathrm{~J} / \mathrm{m}^{2}\right)$ interface. Graphene defects are found to enhance the interface stability especially for the case of divacancy defects resulting in the adhesion energy of $-2.82 \mathrm{~J} / \mathrm{m}^{2}$. On the other hand, $\gamma$-MoC C-terminated (001) supported graphene are less stable with the adhesion energy of $-0.90 \mathrm{~J} / \mathrm{m}^{2}$, and exhibits poor HER activity as an isolated graphene. ${ }^{17}$ 


\section{Hydrogen adsorption and free energies}

The hydrogen adsorption energy $\Delta E_{\mathrm{H}}$ is defined as,

$$
\Delta E_{\mathrm{H}}=E_{\mathrm{H}(n)}-E_{\mathrm{H}(n-1)}-0.5 E_{\mathrm{H}_{2}},
$$

where $E_{\mathrm{H}(n)}$ and $E_{\mathrm{H}(n-1)}$ are the total energies of the system with $n$ and $n-1$ adsorbed hydrogen $\mathrm{H}^{*}$, and $E_{\mathrm{H}_{2}}$ is the energy of $\mathrm{H}_{2}$ molecule. To study the hydrogen adsorption sites on the supported graphene surfaces, we use a single hydrogen atom to probe all possible adsorption sites i.e. for $n=1$. The hydrogen adsorption free energy for the $n^{\text {th }}$ adsorbed hydrogen at standard condition is defined as

$$
\Delta G_{\mathrm{H}}=\Delta E_{\mathrm{H}}+\Delta E_{\mathrm{ZPE}}-T \Delta S,
$$

where $\Delta E_{\mathrm{H}}$ is the hydrogen adsorption energy as defined in Eq. (S2), $\Delta E_{\mathrm{ZPE}}$ is the zero-point energy difference between $\mathrm{H}^{*}$ and $\mathrm{H}_{2}(\mathrm{~g})$. For all systems, we use $\Delta E_{\mathrm{ZPE}}=0.18 \mathrm{eV}$ which is the averaged value obtained from MoC-G and MoC-DV at low coverages. This is obtained by relaxing graphene and the supporting Mo layer. Comparing to metal surfaces $\left(\Delta E_{\mathrm{ZPE}} \cong 0.04 \mathrm{eV}\right)$, this high value is attributed to the high $\mathrm{C}-\mathrm{H}$ bond stretching mode. ${ }^{18}$ The entropy difference $T \Delta S$ between the adsorbed and gas phase $\mathrm{H}_{2}(\mathrm{~g})$ at standard conditions is $-0.20 \mathrm{eV}$ as obtained experimentally. ${ }^{19}$

The total hydrogen adsorption free energy is defined as,

$$
\Delta G_{\text {tot }}=\sum_{1}^{n} \Delta G_{\mathrm{H}}+n|e| U-k_{B} T \ln \left[\mathrm{H}^{+}\right]+\Delta E_{\text {water }}
$$

Here, the adsorbate-water interactions $\Delta E_{\text {water }}$ is verified to be smaller than $\pm 0.1 \mathrm{eV}$, and hence will be ignored. Also, in acidic environment, $-k_{B} T \ln \left[\mathrm{H}^{+}\right]$is negligibly small and will also be ignored.
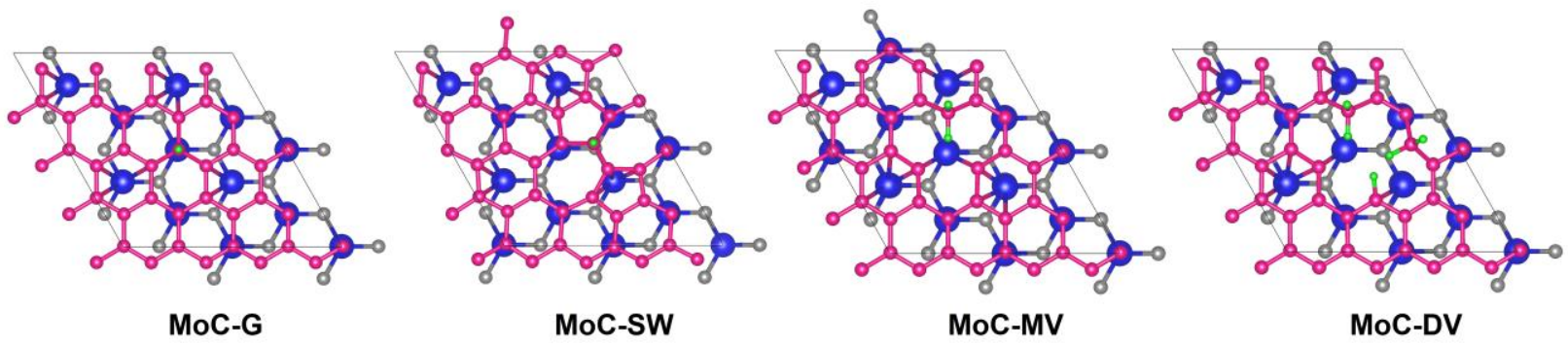

Figure S2. The hydrogen adsorption configurations near the HER reversible potential for the four $y$-MoC supported graphene systems. The carbon atoms of graphene ( $\gamma-\mathrm{MoC})$ are in magenta (gray), and the Mo atoms of $\mathrm{Y}-\mathrm{MoC}$ are in blue. The hydrogen atoms are in green. For the MoC-G, MoC-SW, MoC-MV and MoC-DV, the hydrogen coverages are 1/32 ML, 1/32 ML, 2/31 ML and 5/30 ML, respectively (monolayer, $\mathrm{H}^{*}$ per surface carbon atoms). These hydrogen coverages are presented by the lowest line near the potential of $0 \mathrm{vs}$. SHE in Figure 2(b). 


\section{The NEB calculations of reaction pathways and the extrapolated scheme}

In real electrochemical systems, HER proceeds at a constant potential. However, modeling the reactions using finite-size periodic supercells results in variations of electrostatic potential at solid-water interface along reaction path. Thus, we adopt an extrapolation scheme to correct for activation energy $E_{\text {avt }}$, as can be also done for reaction energy $\Delta E$. For reaction energy $\Delta E\left(U_{\mathrm{SHE}}\right)$ at the HER reversible potential $U_{\mathrm{SHE}}$ we can write that

$$
\Delta E\left(\begin{array}{l}
\Phi_{I S} \\
\Phi_{F S}
\end{array}\right)=\left\{\begin{array}{l}
E_{F S}\left(\Phi_{F S}\right)-E_{I S}\left(\Phi_{I S}\right)+\frac{\left(q_{F S}-q_{I S}\right)\left(\Phi_{F S}-\Phi_{I S}\right)}{2} \\
E_{F S}\left(\Phi_{F S}\right)-E_{I S}\left(\Phi_{I S}\right)-\frac{\left(q_{F S}-q_{I S}\right)\left(\Phi_{F S}-\Phi_{I S}\right)}{2}
\end{array},\right.
$$

where $\Phi_{i}$ is the work function at initial or final state where $i=I S$ or $F S$. The excess charge $q_{i}$ on surface at state $i$ is evaluated by analyzing the amount of charge being oxidized in water layer. $E_{i}\left(\Phi_{i}\right)$ is the total energy of the system at state $i$. In the same manner, to extrapolate activation energy, we can write that

$$
E_{\text {avt }}\left(\begin{array}{l}
\Phi_{I S} \\
\Phi_{T S}
\end{array}\right)=\left\{\begin{array}{l}
E_{T S}\left(\Phi_{T S}\right)-E_{I S}\left(\Phi_{I S}\right)+\frac{\left(q_{T S}-q_{I S}\right)\left(\Phi_{T S}-\Phi_{I S}\right)}{2} \\
E_{T S}\left(\Phi_{T S}\right)-E_{I S}\left(\Phi_{I S}\right)-\frac{\left(q_{T S}-q_{I S}\right)\left(\Phi_{T S}-\Phi_{I S}\right)}{2}
\end{array} .\right.
$$

From Eq. (S5) and Eq. (S6), the values of $\Delta E$ and $E_{\text {avt }}$ with respect to potential are extrapolated by the two states of FS/TS and as shown in Figure $S 2$. The values correspond to the standard electrode potential $U_{\mathrm{SHE}}$ are listed in Table S2 for the water layers in H-up and H-down configurations, respectively. From Eq. (S6), we can obtain the change in $E_{\text {avt }}$ with respect to work function

$$
E_{a v t}\left(\Phi_{T S}\right)-E_{a v t}\left(\Phi_{I S}\right)=-\Delta q\left(\Phi_{T S}-\Phi_{I S}\right)
$$

where $-\Delta q$ is in analogues to symmetry factor $\beta=-\Delta q$.

Table S2. $\Delta E$ and $E_{\text {avt }}$ (in eV) for the three HER elementary steps at $U_{\mathrm{SHE}}$ obtained from the model with water

\begin{tabular}{|c|c|c|c|c|c|c|c|c|c|}
\hline \multirow[t]{2}{*}{ H-up } & \multicolumn{3}{|c|}{ Volmer } & \multicolumn{3}{|c|}{ Heyrovsky } & \multicolumn{3}{|c|}{ Tafel } \\
\hline & $\Delta E$ & $E_{a v t}$ & $\boldsymbol{\beta}$ & $\Delta E$ & $E_{a v t}$ & $\boldsymbol{\beta}$ & $\Delta E$ & $E_{a v t}$ & $\boldsymbol{\beta}$ \\
\hline MoC-G & 0.46 & 0.40 & 0.07 & 0.60 & 0.85 & 0.12 & 0.33 & 2.98 & 0 \\
\hline MoC-SW & 0.30 & 0.27 & 0.04 & 0.82 & 1.32 & 0.19 & 0.63 & 2.58 & 0 \\
\hline MoC-MV & 0.30 & 0.30 & 0.07 & 0.96 & 1.62 & 0.29 & 0.89 & 2.70 & 0 \\
\hline MoC-DV & 0.66 & 0.50 & 0.10 & 0.33 & 1.29 & 0.28 & 0.51 & 2.42 & 0 \\
\hline \multirow[t]{2}{*}{ H-down } & \multicolumn{3}{|c|}{ Volmer } & \multicolumn{3}{|c|}{ Heyrovsky } & \multicolumn{3}{|c|}{ Tafel } \\
\hline & $\Delta E$ & $E_{a v t}$ & $\boldsymbol{\beta}$ & $\Delta E$ & $E_{a v t}$ & $\boldsymbol{\beta}$ & $\Delta E$ & $E_{a v t}$ & $\beta$ \\
\hline MoC-G & 0.04 & 0.46 & 0.14 & 0.28 & 0.94 & 0.20 & 0.33 & 2.98 & 0 \\
\hline MoC-SW & 0.43 & 0.23 & 0.00 & 1.24 & 1.45 & 0.27 & 0.63 & 2.58 & 0 \\
\hline MoC-MV & 0.61 & 0.16 & 0.10 & 1.06 & 1.58 & 0.32 & 0.89 & 2.70 & 0 \\
\hline MoC-DV & 0.45 & 0.45 & 0.13 & 0.29 & 0.69 & 0.30 & 0.51 & 2.42 & 0 \\
\hline
\end{tabular}
layer in $\mathrm{H}$-up and $\mathrm{H}$-down configuration. We also list the symmetry factor $\beta$ of the Volmer and Heyrovsky reaction. Note that $\beta=0$ for the Tafel reaction as there is no charge transfer between water layer and surface during reaction. 

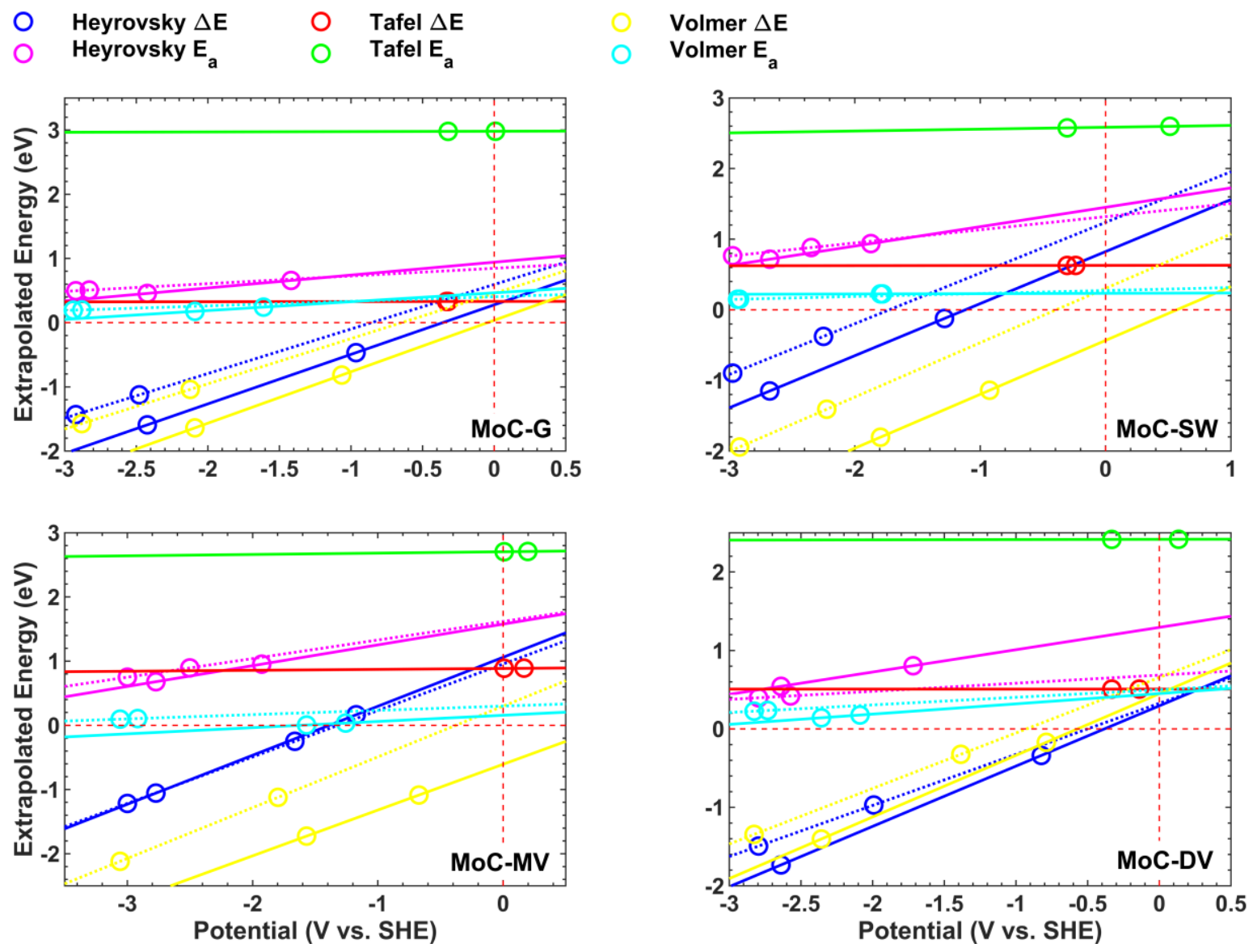

Figure S3. The extrapolation scheme of the $\mathrm{Y}-\mathrm{MoC}$ supported graphene systems. The circles represent the total energies of the initial and final states for $\Delta E$ and of the initial and transition states for $E_{a v t}$. The results obtained using the $\mathrm{H}$-up and $\mathrm{H}$-down water layer models are represented by the dashed and the solid lines, respectively.

\section{The application of thermodynamic exchange current model}

Using the definition of reversible hydrogen electrode, $G_{\mathrm{H}^{+}}=|e| U_{\mathrm{SHE}}+\frac{1}{2} G_{\mathrm{H}_{2}}$, we can write the standard free energy of the Volmer reaction, $2 \mathrm{H}^{+}(a q)+2 e^{-} \rightarrow \mathrm{H}^{+}(a q)+e^{-}+\mathrm{H}^{*}$, as

$$
\begin{aligned}
\Delta G^{0} & =\left(G_{\mathrm{H}^{*}}-|e| U+G_{\mathrm{H}^{+}}\right)-\left(2 G_{\mathrm{H}^{+}}-2|e| U\right) \\
& =G_{\mathrm{H}^{*}}+|e|\left(U-U_{\mathrm{SHE}}\right)-\frac{1}{2} G_{\mathrm{H}_{2}} .
\end{aligned}
$$

Using the definition of hydrogen adsorption free energy as defined in the main text,

$$
\Delta G_{\mathrm{H}}=G_{\mathrm{H}^{*}}-\frac{1}{2} G_{\mathrm{H}_{2}},
$$

we can get

$$
\Delta G^{0}=\Delta G_{\mathrm{H}}+|e| \eta,
$$

where $\eta=U-U_{\mathrm{SHE}}$. At equilibrium, $\eta=0$, the total free energy is zero that $0=\Delta G_{\mathrm{H}}+k_{B} T \ln \left(K_{V}\right)$, which leads to 


$$
K_{V}=\exp \left(-\frac{\Delta G_{\mathrm{H}}}{k_{B} T}\right)
$$

The forward and the backward rate of the Volmer reaction are

$$
r=\left\{\begin{array}{l}
k_{V}^{0}(1-\theta) \exp (-\alpha f \eta) \\
k_{-V}^{0} \theta \exp [(1-\alpha) f \eta]
\end{array}\right.
$$

At equilibrium, the forward and the backward rates are equal, we can write that $\frac{k_{V}^{0}(1-\theta) \exp (-\alpha f \eta)}{k_{-V}^{0} \theta \exp [(1-\alpha) f \eta]}=1$ or

$$
\theta=\frac{K_{V}}{\exp (\eta f)+K_{V}}
$$

where $K_{V}=\frac{k_{V}^{0}}{k_{-V}^{0}}$ for $\Delta G_{\mathrm{H}}>0$. If we define that $K_{V}=\frac{k_{-V}^{0}}{k_{V}^{0}}$ for $\Delta G_{\mathrm{H}}<0$, we get the other expression that

$$
\theta=\frac{1}{K_{V} \exp (\eta f)+1}
$$

This expression of $\theta$ is also obtained by Shinagawa et. al using a theoretical approach. ${ }^{20}$ It follows a Langmuir isotherm which is conditioned to three requirements: 1) there is only a monolayer of adsorption sites, 2) the $\mathrm{H}^{*}-\mathrm{H}^{*}$ interactions are minimal compare to surface $-\mathrm{H}^{*}$ interactions, 3) the adsorption sites are identical, and 4) each adsorption site cannot be occupied by more than one $\mathrm{H}^{*}$. Norskov et. al. applied such expression of $\theta(\eta=0)$ to study the thermodynamic exchange currents (defined at zero overpotential) on metal surfaces ${ }^{21}$. These metal surfaces satisfy the requirements: the adsorption sites on metal surfaces are identical with a nearly constant $\Delta G_{\mathrm{H}}$ below and at $\theta=1$, which shows negligible $\mathrm{H}^{*}-\mathrm{H}^{*}$ interactions. However, the $\Delta G_{\mathrm{H}}$ sharply increases above $\theta=1$, which indicates that all the adsorption sites are fully occupied. ${ }^{22}$

Table S3. The averaged $\Delta G_{\mathrm{H}}$ used for the exchange current model of Eq. (E) and the

\begin{tabular}{lcc} 
computed $j_{0}$ & & \\
\multicolumn{1}{c}{ Systems } & $\boldsymbol{\Delta} \boldsymbol{G}_{\mathbf{H}}$ & $\boldsymbol{j}_{\mathbf{0}}$ \\
MoC-G & 0.21 & $-1.14 \times 10^{-6}$ \\
MoC-SW & -0.27 & $-8.92 \times 10^{-8}$ \\
MoC-MV & 0.21 & $-5.78 \times 10^{-6}$ \\
MoC-DV & 0.10 & $-3.34 \times 10^{-4}$
\end{tabular}

We claim that this $\theta$ model can also be applied on $\gamma$-MoC supported graphene systems. For MoC$\mathrm{G}$ and MoC-SW, we find that a constant $\Delta G_{\mathrm{H}}=0.2 \mathrm{eV}$ or $-0.2 \mathrm{eV}$ under 1/32 ML (monolayer, $\mathrm{H}^{*}$ per surface carbon atoms), respectively, and $\Delta G_{\mathrm{H}}$ sharply increases above 1/32 ML. For MoC-MV and MoC$\mathrm{DV}, \Delta G_{\mathrm{H}}$ wiggles with hydrogen coverage, as can be seen in Figure $2 \mathrm{a}$. However, if we remove the strong adsorption sites, i.e. where the last $\mathrm{H}^{*}$ occupied at 2/31 ML for MoC-MV, or 1/30, 2/30 ML and 5/30 ML for MoC-DV, we can see the same trend (constant $\Delta G_{\mathrm{H}}$ vs hydrogen coverage) as discussed. We can ignore these stronger adsorption sites because $\mathrm{H}^{*}$ adsorbed at these coverages are less likely to be involved in HER than the other sites. Therefore, to compute the exchange current using the thermodynamic exchange current model, we use the averaged $\Delta G_{\mathrm{H}}$ at the region where $\Delta G_{\mathrm{H}}$ is nearly constant with increasing hydrogen coverage: 1/32 ML for MoC-G and MoC-SW, and (1, 3, 4, 5, 6, 7)/31 ML and (3, 4, 5, 6, 7)/30 ML, for MoC-MV and MoC-DV, respectively. 
Eq. (2) in the main text, $j=n F k_{0} C_{t o t} \theta \exp (-\beta f \eta)$, can be written in the Butler-Volmer form as $j=j_{0} \exp (-\beta f \eta)$, where $j_{0}$ is a constant called exchange current density. At $\eta=0$ where $j_{0}$ is defined, we can use the averaged $\Delta G_{\mathrm{H}}$ to compute $j_{0}$. The results are shown in Table S3.

\section{Experimental fitting of $\boldsymbol{k}_{\mathbf{0}}$}

The value of $k_{0}$ for the $\gamma$-MoC supported graphene systems is determined by fitting the experimental values with the current model of Eq. (2) and (3) on graphene coated $\mathrm{Mo}_{\mathrm{x}} \mathrm{C}$ systems in experiments. ${ }^{23-25}$ All the experimental currents are perfectly fitted with the model using $\beta$ in the range of 0.3 and 0.4 . Note here that the overall curvature of the experimental currents is controlled by $\beta$ and is independent to $k_{0}$. We show the data in Table S4 below. All the currents are fitted with $\mathrm{R}^{2}=0.99$.

Table S4. The $k_{0}$ fitted from experimental values by choosing $\beta$ in the range of 0.3 and 0.4 .

\begin{tabular}{ccc}
\hline EXP. 1 (Ref. 23, Fig. 4a) & $\boldsymbol{\beta}$ & $\boldsymbol{k}_{\mathbf{0}}$ \\
$\mathrm{Mo}_{2}$ C/NC750 & 0.3 & 168 \\
$\mathrm{Mo}_{2}$ C/NCS750 & 0.4 & 189 \\
$\mathrm{Mo}_{2}$ C/G3-NCS750 & 0.4 & 295 \\
EXP. 2 (Ref. 24, Fig. 4a) & & \\
MoCx@C-1 & 0.3 & 214 \\
EXP. 3 (Ref. 25, Fig. 5a) & & \\
1.1g & 0.3 & 162 \\
2.2g & 0.3 & 263 \\
3.3g & 0.4 & 383
\end{tabular}

\section{Theoretical derivation of the Tafel Slope}

From Eq. (2) and Eq. (3), we obtain:

$$
j= \begin{cases}n F k_{0} C_{\text {tot }} \frac{1}{K_{V} \exp (\eta f)+1} \exp (-\beta f \eta) & \text { for } \Delta G_{\mathrm{H}}<0 \\ n F k_{0} C_{t o t} \frac{K_{V}}{\exp (\eta f)+K_{V}} \exp (-\beta f \eta) & \text { for } \Delta G_{\mathrm{H}}>0\end{cases}
$$

Near the zero overpotential; $K_{V} \exp (\eta f) \gg 1$ for $\Delta G_{\mathrm{H}}<0$ and $\exp (\eta f) \gg K_{V}$ for $\Delta G_{\mathrm{H}}>0$, we can rewrite that

$$
\begin{gathered}
j(\eta \approx 0) \cong\left\{\begin{array}{cc}
n F k_{0} C_{t o t} \frac{1}{K_{V} \exp (\eta f)} \exp (-\beta f \eta) & \text { for } \Delta G_{\mathrm{H}}<0 \\
n F k_{0} C_{t o t} \frac{K_{V}}{\exp (\eta f)+K_{V}} \exp (-\beta f \eta) & \text { for } \Delta G_{\mathrm{H}}>0 \\
\text { or } & \text { for } \Delta G_{\mathrm{H}}<0
\end{array}\right. \\
\log (j(\eta \approx 0)) \cong\left\{\begin{array}{cc}
\log \left(n F k_{0} C_{t o t}\right)-\log \left(K_{V}\right)-\frac{1}{2.303} \eta f+\frac{1}{2.303}(-\beta f \eta) & \text { for } \Delta G_{\mathrm{H}}>0 \\
\log \left(n F k_{0} C_{t o t}\right)+\log \left(K_{V}\right)-\frac{1}{2.303} \eta f+\frac{1}{2.303}(-\beta f \eta) &
\end{array}\right.
\end{gathered}
$$

Taking the derivative with respect to $\eta$ for both cases, we can obtain 


$$
\frac{d \log (j(\eta \approx 0))}{d \eta} \cong-\frac{1}{2.303} f(\beta+1)
$$

The Tafel slope is defined as $d \eta / d \log (j)$, therefore, near $\eta \approx 0$,

$$
\frac{d \eta}{d \log (j(\eta \approx 0))}=-\frac{2.303 R T}{F(\beta+1)}
$$

\section{References}

1. Kresse, G.; Hafner, J., Ab initio molecular dynamics for liquid metals. Phys. Rev. B 1993, 47, 558561.

2. Kresse, G.; Hafner, J., Ab initio molecular dynamics for open-shell transition metals. Physical Review B 1993, 48, 13115-13118.

3. Kresse, G.; Hafner, J., Ab-initio molecular-dynamics simulation of the liquid-metal amorphoussemiconductor transition in germanium Phys. Rev. B 1994, 49, 14251-14269.

4. Kresse, G.; Joubert, D., From ultrasoft pseudopotentials to the projector augmented-wave method. Physical Review B 1999, 59, 1758-1775.

5. Tkatchenko, A.; Scheffler, M., Accurate Molecular Van Der Waals Interactions from GroundState Electron Density and Free-Atom Reference Data. Physical review letters 2009, 102, 073005.

6. Henkelman, G.; Uberuaga, B. P.; Jónsson, H., A climbing image nudged elastic band method for finding saddle points and minimum energy paths. The Journal of Chemical Physics 2000, 113, 9901-9904. 7. Rossmeisl, J.; Skúlason, E.; Björketun, M. E.; Tripkovic, V.; Nørskov, J. K., Modeling the electrified solid-liquid interface. Chemical Physics Letters 2008, 466, 68-71.

8. Eigen, M., Proton transfer, acid-base aatalysis, and enzymatic hydrolysis. Angew Chem Int Ed Eng/ 1964, 3, 72.

9. $\quad$ Ogasawara, H.; Brena, B.; Nordlund, D.; Nyberg, M.; Pelmenschikov, A.; Pettersson, L. G.; Nilsson, A., Structure and bonding of water on Pt(111). Phys Rev Lett 2002, 89, 276102.

10. Hugosson, H. W.; Eriksson, O.; Nordström, L., Theory of phase stabilities and bonding mechanisms in stoichiometric and substoichiometric molybdenum carbide. J. Appl. Phys. 1999, 86.

11. KuO, K.; HÄGG, G., A New Molybdenum Carbide. Nature 1952, 170.

12. Wan, C.; Regmi, Y. N.; Leonard, B. M., Multiple phases of molybdenum carbide as electrocatalysts for the hydrogen evolution reaction. Angew Chem Int Ed Eng/ 2014, 53, 6407-10.

13. Vines, F.; Sousa, C.; Liu, P.; Rodriguez, J. A.; Illas, F., A systematic density functional theory study of the electronic structure of bulk and (001) surface of transition-metals carbides. J Chem Phys 2005, 122, 174709.

14. Politi, J. R.; Vines, F.; Rodriguez, J. A.; Illas, F., Atomic and electronic structure of molybdenum carbide phases: bulk and low Miller-index surfaces. Phys Chem Chem Phys 2013, 15, 12617-25.

15. Zur, A.; McGill, T. C., Lattice match: An application to heteroepitaxy. Journal of applied physics 1984, 55, 378-386.

16. Gao, W.; Xiao, P.; Henkelman, G.; Liechti, K. M.; Huang, R., Interfacial adhesion between graphene and silicon dioxide by density functional theory with van der Waals corrections. Journal of Physics D: Applied Physics 2014, 47.

17. Tsai, C.; Abild-Pedersen, F.; Nørskov, J. K., Tuning the MoS2 Edge-Site Activity for Hydrogen Evolution via Support Interactions. Nano Lett. 2014, 14, 7.

18. Deng, Y.; Mou, J.; Wu, H.; Zhou, L.; Zheng, Q.; Lam, K. H.; Xu, C.; Lin, D., Enhanced Electrochemical Performance in Ni-Doped LiMn2O4-Based Composite Cathodes for Lithium-lon Batteries. ChemElectroChem 2017, 4, 1362-1371. 
19. Chase, M. W.; Curnutt, J. L.; Downey, J. R.; McDonald, R. A.; Syverud, A. N.; Valenzuela, E. A., JANAF Thermochemical Tables, 1982 Supplement. Journal of Physical and Chemical Reference Data 1982, 11, 695.

20. Shinagawa, T.; Garcia-Esparza, A. T.; Takanabe, K., Insight on Tafel slopes from a microkinetic analysis of aqueous electrocatalysis for energy conversion. Sci Rep 2015, 5, 13801.

21. Nørskov, J. K.; Bligaard, T.; Logadottir, A.; Kitchin, J. R.; Chen, J. G.; Pandelov, S.; Stimming, U., Trends in the Exchange Current for Hydrogen Evolution. Journal of The Electrochemical Society 2005, 152.

22. Skúlason, E.; Tripkovic, V.; Björketun, M. E.; Gudmundsdóttir, S.; Karlberg, G.; Rossmeisl, J.; Bligaard, T.; Jónsson, H.; Nørskov, J. K., Modeling the Electrochemical Hydrogen Oxidation and Evolution Reactions on the Basis of Density Functional Theory Calculations. The Journal of Physical Chemistry $C$ 2010, 114, 18182-18197.

23. Wei, H.; Xi, Q.; Chen, X.; Guo, D.; Ding, F.; Yang, Z.; Wang, S.; Li, J.; Huang, S., Molybdenum Carbide Nanoparticles Coated into the Graphene Wrapping N-Doped Porous Carbon Microspheres for Highly Efficient Electrocatalytic Hydrogen Evolution Both in Acidic and Alkaline Media. Adv Sci (Weinh) 2018, 5, 1700733.

24. Yang, X.; Feng, X.; Tan, H.; Zang, H.; Wang, X.; Wang, Y.; Wang, E.; Li, Y., N-Doped graphenecoated molybdenum carbide nanoparticles as highly efficient electrocatalysts for the hydrogen evolution reaction. Journal of Materials Chemistry A 2016, 4, 3947-3954.

25. Li, J. S.; Wang, Y.; Liu, C. H.; Li, S. L.; Wang, Y. G.; Dong, L. Z.; Dai, Z. H.; Li, Y. F.; Lan, Y. Q., Coupled molybdenum carbide and reduced graphene oxide electrocatalysts for efficient hydrogen evolution. Nat Commun 2016, 7, 11204. 